\title{
Cluster observation of magnetohydrodynamic turbulence in the plasma sheet boundary layer
}

\author{
Y. Narita ${ }^{1,2^{*}}$
}

\begin{abstract}
Measurement of turbulent magnetic field is presented from the Earth magnetotail crossing of the Cluster spacecraft on August 25, 2006, as an ideal case study of magnetohydrodynamic turbulence in the plasma sheet boundary layer on a spatial scale of about $10,000 \mathrm{~km}$. The fluctuation energy of the magnetic field is evaluated in both the frequency and wavevector domains. The observed plasma sheet turbulence event shows anisotropy in the wavevector domain with a spectral extension perpendicular to the mean magnetic field. The analyses of the dispersion relation and phase speed diagrams indicate that the coherent wave components should be regarded as a set of the linear-mode waves and the other fluctuation components in magnetohydrodynamics. Although the magnetic field fluctuation amplitudes are sufficiently small compared to the large-scale field strength, there is no clear indication of the linear-mode dominance in the plasma sheet. As a lesson, magnetohydrodynamic turbulence must be modeled by including both linear-mode waves and nonlinear wave components such as sideband waves.
\end{abstract}

Keywords: Wavevector-frequency spectra, Magnetohydrodynamic turbulence, Plasma sheet boundary layer, Geomagnetic tail

\section{Introduction}

Plasma sheet surrounding a current sheet occur in many places in space plasmas such as planetary magnetospheres, heliosphere, and solar atmosphere, whenever a shear or anti-parallel magnetic field geometry appears. The plasma sheet, together with the current sheet, sustains anti-parallel magnetic fields through Ampère's law. It has been pointed out that the plasma sheet develops into turbulence (Vörös et al. 2004, 2007a, b). Earlier measurements have indicated the existence of turbulence or turbulent fluctuations in the plasma sheet. The ISEE-3 observations show turbulent activities in the distant geomagnetic tail from 60 to $240 R_{\mathrm{e}}$ (Tsurutani et al. 1984), where $1 R_{e}=6378 \mathrm{~km}$ is the Earth radius, and a broadband spectrum which extends to a power-law spectrum at frequencies higher than $0.1 \mathrm{~Hz}$ with both incompressible

\footnotetext{
*Correspondence: yasuhito.narita@oeaw.ac.at

1 Space Research Institute, Austrian Academy of Sciences, Schmiedlstraße 6, 8042 Graz, Austria

Full list of author information is available at the end of the article
}

(like circularly polarized, parallel-propagating waves) and compressible fluctuations (Tsurutani et al. 1986). Also, ISEE-3 data show that variations of the magnetic field in short period (less than $1 \mathrm{~min}$ ) increase with higher geomagnetic activity (Tsurutani et al. 1987). On spatial scales larger than ion gyro-radius, the disturbance of the plasma and the magnetic field may be regarded as a realization of magnetohydrodynamics (MHD) turbulence. The current sheet is a very thin region where the magnetic field reverses direction, and is typically surrounded on both sides by a much wider plasma sheet, e.g., in the Earth distant tail (Tsurutani et al. 1984a, c; Baumjohann et al. 1989) and in the heliospheric current sheet (Smith et al. 1978; Winterhalter et al. 1994). The magnetic field decrease is caused by a diamagnetic effect from the hot plasma in the plasma sheet.

Many ideas and models have been proposed to describe MHD turbulence, e.g., phenomenological models for isotropic turbulence (Iroshnikov 1964; Kraichnan 1965) and anisotropic turbulence (Goldreich and Sridhar 1995, 1997), closure theory of MHD turbulence (Yokoi et al. 
2008), and field-theoretic perturbative theories (Kaburaki and Uchida 1971; Verma 2004). Above all, it is essential to know how the wavevector anisotropy is organized in the energy spectrum (which indicates the sense of energy cascade) and if the turbulent fluctuations follow dispersion relations for the linear modes.

The goal of this manuscript is to observationally evaluate the spatio-temporal properties of magnetohydrodynamic turbulence in the plasma sheet boundary layer in the Earth magnetotail at a distance of about $16 R_{e}$ such as the energy spectrum and the wave characteristics (linear modes or sideband waves, dispersion relations, propagation directions, and phase speed distribution) using the measurement by the Cluster mission (Escoubet et al. 2001). As a result, constraints on MHD turbulence in the plasma sheet boundary layer are given such that not only the linear-mode waves but also sideband wave component needs to be included in constructing or improving MHD turbulence theories.

\section{Magnetotail crossing}

Cluster observation on August 25, 2006, 1300-1500 UT is selected for the study of MHD turbulence in the plasma sheet boundary layer. This interval represents the longest interval of the Cluster observation in the Earth magnetotail plasma sheet. Figure 1 displays the time series data

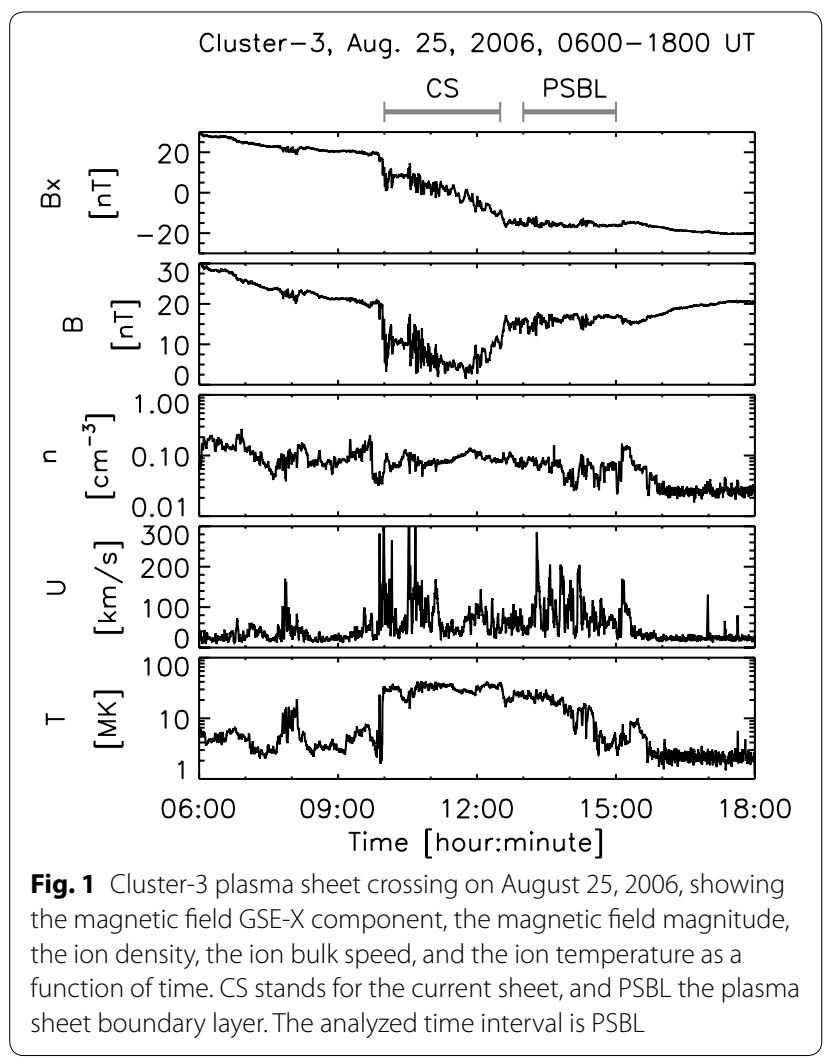

of the sunward component (GSE-X) and the magnitude of the magnetic field, the ion density, the ion bulk speed, and the ion temperature measured by Cluster- 3 fluxgate magnetometer (Balogh et al. 2001) and ion spectrometry (Rème et al. 2001) along its trajectory from the northern lobe (0600-1000 UT) to the plasma sheet (1000-1500 UT) and further to the southern lobe region (1500-1800 UT). The interval 1000-1230 UT represents a crossing of the current sheet with a decrease of the magnetic field magnitude and a shear in the sunward component. The interval 1300-1500 UT shows a crossing of the outer part of the plasma sheet with a nearly constant magnetic field (Fig. 2) and represents a plasma sheet boundary layer with an about $10 \%$ decrease from the magnetic field magnitude in the lobe region.

The interval 1300-1500 UT is chosen for a test of magnetohydrodynamic turbulence against the Cluster data. The four Cluster spacecraft form a nearly regular tetrahedron with the configuration index $Q_{\mathrm{G}}$ close 3 (Robert et al. 2001; von Stein et al. 1992). The inter-spacecraft distance is about $10,000 \mathrm{~km}$, which is about 10 times larger than the local ion gyro-radius and ideal testing for MHD turbulence in the plasma sheet. The mean values and the root-mean-square values of the magnetic field and the ion velocity moments are summarized in Table 1 . In Fig. 1, from 1000 to $1230 \mathrm{UT}$, the magnetic field $B_{\mathrm{x}}$ component changes from a positive value to a negative one. This transition is identified as the current sheet. A statistical study on the current sheet shows that there are different types of the current sheets. Some have a width of below $2000 \mathrm{~km}$ and the others a width of about $4000 \mathrm{~km}$ (Runov et al. 2006). From 1000 to 1230 UT on the analyzed interval, Cluster cross a distance of about 10,250 $\mathrm{km}$ in the GSE-Z direction. Therefore, this interval represents a thick current sheet event. Also coincident with this field rotation there is a large magnetic field decrease. This is identified as the plasma sheet. In the case of the analyzed interval, the width of the plasma sheet is nearly the same as the current sheet.

The plasma sheet boundary layer has been noted to have distinct magnetosonic mode waves generated by energetic ion beams during intervals of magnetic reconnection in the distant tail (Cowley et al. 1984; Tsurutani and Smith 1984; Tsurutani et al. 1985; Gary et al. 1985). The interval of the plasma sheet crossing is associated with geomagnetically quiet one or only moderately active. The AE index (provisional data) reaches $101 \mathrm{nT}$ (hourly averaged) at 1400 $\mathrm{UT}$, and otherwise the index is below $100 \mathrm{nT}$ on August 25, 2006. The ion spectrogram at Cluster- 3 shows that there are two ion populations: the main component associated with the bursty bulk flows at an energy of 1-10 keV and the other component with a lower density and a lower energy of about $100 \mathrm{eV}$ in the tailward direction. 


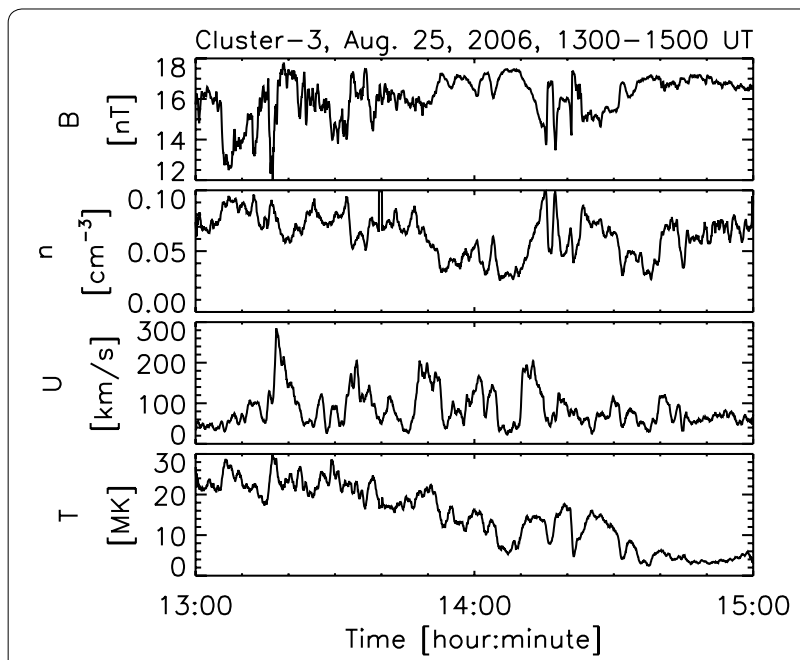

Fig. 2 Magnified view of the fluctuations of the magnetic field magnitude, the ion density, the ion bulk speed, and the ion temperature from Cluster-3 from 1300 to 1500 UT for the data shown in Fig. 1

Table 1 Mean values (denoted by the angular bracket $\langle\cdots\rangle)$ and root-mean-square (denoted by $\sigma(\cdots))$ of the magnetic field $\vec{B}$, the ion bulk velocity $\vec{U}$, the ion number density $n$, and the ion temperature $T$ on the interval 1300 to 1500 UT on August 25, 2006 (Cluster-3 data)

\begin{tabular}{llll}
\hline$\left\langle B_{x}\right\rangle,\left\langle B_{y}\right\rangle,\left\langle B_{z}\right\rangle$ & $-15.66 \mathrm{nT}$ & $-2.31 \mathrm{nT}$ & $2.01 \mathrm{nT}$ \\
$\langle|\vec{B}|\rangle$ & $16.10 \mathrm{nT}$ & & \\
$\left\langle U_{x}\right\rangle,\left\langle U_{y}\right\rangle,\left\langle U_{z}\right\rangle$ & $25.27 \mathrm{~km} / \mathrm{s}$ & $9.05 \mathrm{~km} / \mathrm{s}$ & $12.89 \mathrm{~km} / \mathrm{s}$ \\
$\langle|\vec{U}|\rangle$ & $85.76 \mathrm{~km} / \mathrm{s}$ & & \\
$\langle n\rangle$ & $0.064 \mathrm{~cm}^{-3}$ & & \\
$\left\langle T_{\|}\right\rangle,\left\langle T_{\perp}\right\rangle$ & $18.88 \mathrm{MK}$ & $12.38 \mathrm{MK}$ & \\
$\langle T\rangle$ & $14.54 \mathrm{MK}$ & & \\
$\sigma\left(B_{x}\right), \sigma\left(B_{\mathrm{y}}\right), \sigma\left(B_{z}\right)$ & $0.97 \mathrm{nT}$ & $0.92 \mathrm{nT}$ & $1.98 \mathrm{nT}$ \\
$\sigma(|\vec{B}|)$ & $1.03 \mathrm{nT}$ & & \\
$\sigma\left(U_{x}\right), \sigma\left(U_{\mathrm{y}}\right), \sigma\left(U_{z}\right)$ & $81.27 \mathrm{~km} / \mathrm{s}$ & $39.00 \mathrm{~km} / \mathrm{s}$ & $29.12 \mathrm{~km} / \mathrm{s}$ \\
$\sigma(|\vec{U}|)$ & $50.01 \mathrm{~km} / \mathrm{s}$ & & \\
$\sigma(n)$ & $0.026 \mathrm{~cm}{ }^{-3}$ & & \\
$\sigma\left(T_{\|}\right), \sigma\left(T_{\perp}\right)$ & $11.26 \mathrm{MK}$ & $7.14 \mathrm{MK}$ & \\
$\sigma(T)$ & $7.76 \mathrm{MK}$ & &
\end{tabular}

The magnetic field and the bulk velocities are represented in the GSE coordinate system

As the first step in the turbulence study, the energy spectrum is evaluated for the magnetometer data in the spacecraft-frame frequency domain for the interval 1300-1500 UT (Fig. 3) using the fast Fourier transform algorithm. Both the compressible and incompressible fluctuation powers ( $P_{\text {para }}$ and $P_{\text {perp }}$, respectively) are in comparable order at low frequencies up to $4 \times 10^{-3} \mathrm{~Hz}$. Computation of the two fluctuation powers is easily done in the mean-field-aligned (MFA) coordinate system with

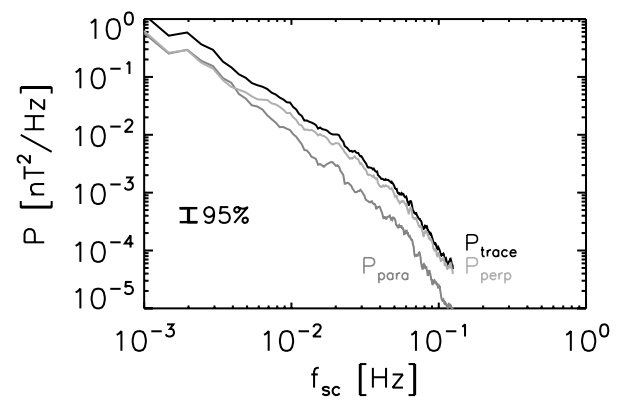

Fig. 3 Magnetic energy spectrum in the spacecraft-frame frequency domain for the trace of cross-spectral density matrix (in black), and the parallel (in dark gray) and the perpendicular (in thin gray) fluctuations to the large-scale magnetic field direction. The spectral curves are obtained on the time interval 1300-1500 UT and are averaged over four spacecraft of Cluster

the $z$ axis in the direction of the mean magnetic field and the $x-z$ plane containing the GSE-X direction, and is tractable to see whether the plasma and the associated fluctuations are treated as incompressible.

The incompressible power $P_{\text {perp }}$ dominates the spectrum above that frequency. The total fluctuation power $\left(P_{\text {trace }}=P_{\text {perp }}+P_{\text {para }}\right)$ shows a power law with an index close to $-5 / 3$ up to $7 \times 10^{-2} \mathrm{~Hz}$ and breaks into a steeper spectrum at higher frequencies. Naively speaking, the formation of the power-law spectrum with an index of $-5 / 3$ and the dominance of incompressible fluctuations in the spectrum are reminiscent of Alfvén waves as the primary fluctuation components of the observed plasma sheet turbulence. This expectation can be examined in more detail using the full potential of the four-point measurements in space as follows.

\section{Multi-point turbulence analysis}

The Multi-point Signal Resonator (MSR) algorithm is used in the analysis (Narita et al. 2011). Various methods have been proposed to detect wave modes through wave vector analysis: six-component method (Storey and Lefeuvre 1979, 1980), eigenvalue and eigenvector decomposition (Schmidt 1986) and its generalization to the problem to the unknown numbers of signals (Choi et al. 1993), minimum variance projection (Neubauer and Glassmeier 1990; Pinçon and Lefeuvre 1991; Motschmann et al. 1995, 1996; Glassmeier et al. 2001). MSR is unique in that it combines two distinct projection methods from spatial coordinates into wavevectors. The first projection is the wave telescope algorithm which is the maximum likelihood method for a Gaussian likelihood function. The second projection is Multiple Signal Classification (MUSIC), which is an eigenvector analysis method. The wave telescope method is an application 
of the minimum variance estimator [also called the least square estimator or the Capon estimator (Capon 1969)] to the four-spacecraft measurements in space. The method is a projection of the vectorial quantity (e.g., magnetic field) sampled at four spatial points from the spatial coordinates onto the three-dimensional wavevector domain (Glassmeier et al. 2001) The method plays a role of the Fourier transform to the wavevector domain. In contrast to the Fourier transform, the wave telescope method estimates the fluctuation amplitudes in the wavevector domain by fitting with a set of plane waves under the constraint of minimizing the isotropic noise in the data. Mathematically, the wave telescope method or the minimum variance estimator is regarded as the maximum likelihood method assuming a Gaussian shape for the likelihood function. The effect of the finite noise is eliminated by employing an extended form of the MUSIC algorithm (Schmidt 1986) and coupling it to the wave telescope projection. The MSR method was developed particularly for studying waves and turbulence using four-point magnetic field data and makes extensive use of the 12-by-12 covariance matrix (three components of the magnetic field measured at four spacecraft) by combining the minimum variance projection with the MUSIC algorithm. The MSR technique is based on the assumption that the measured fluctuations represent a set of plane waves (both coherent and incoherent wave components) and that the fluctuations contain small-amplitude isotropic noise. The energy spectrum is obtained as a function of spacecraft-frame frequencies and wavevectors. The 12-by-12 matrix is from the magnetic field measurements. Therefore, electrostatic fluctuations cannot be studied, which is a weakness. However, degeneration due to the multiple waves at one frequency does not apply here because the 12-by-12 matrix (which is determined in the frequency domain) is projected onto the 3-D wavevector domain, which is a strength of the method.

The multi-point turbulence analysis includes three methods: (1) energy spectrum in the wavevector-frequency domain, (2) dispersion relation diagram (wavenumber dependence of frequencies and propagation angles), and (3) phase speed diagram for coherent wave components. The spectral analysis (method 1) uses both coherent and incoherent wave components, and the wavenumber dependence and the phase speed analyses (methods 2 and 3, respectively) use the coherent wave components. Here, the coherent wave components are defined as fluctuations for local peaks of the energy spectrum in the three-dimensional wavevector domain, and the incoherent components as the other fluctuations. For example, Fig. 4 shows a reduced spectrum obtained by selecting the maximum fluctuation energy over the solid angles as a function of the wavenumbers (or wavevector

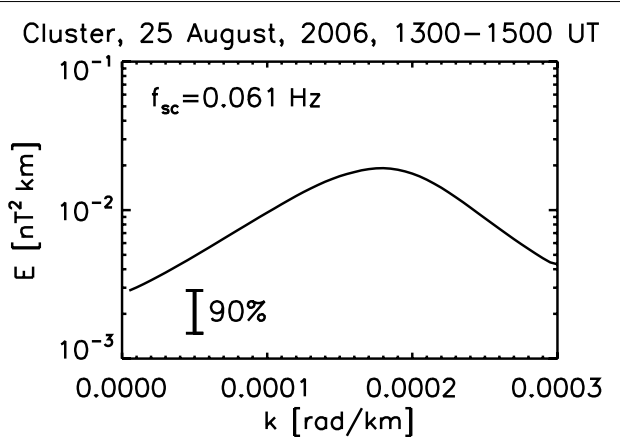

Fig. 4 Energy spectrum in the wavenumber (the magnitude of the wavevector) domain obtained by the MSR technique at a spacecraftframe frequency of $0.0611 \mathrm{~Hz}$. The maximum energy over the wavevector shell $|\vec{k}|$ is plotted

magnitudes) at a frequency of $6.1 \times 10^{-2} \mathrm{~Hz}$. The peak wavenumber of the reduced spectrum is regarded as a coherent wave component and is registered into the database for the coherent wave analysis.

\section{Energy spectrum}

The spectrum estimated by the MSR algorithm is an energy distribution in the four-dimensional spectral domain (as a function of the frequencies and the three components of the wavevectors). The spectrum is visualized in two different ways to reduce onto two-dimensional spectra. First, the spectrum is reduced by slicing along the sunward, the dawn-to-dusk, and the northward directions in the GSE (geocentric-solar-ecliptic) system as a function of the wavevector components and the frequencies (top panels in Fig. 5), which is suited to test for the wavenumber-frequency dependence in search of an organization like dispersion relations. Most of the fluctuation energy is limited to low frequencies irrespective of the wavevectors (below $0.05 \Omega_{\mathrm{p}}$, where $\Omega_{\mathrm{p}}$ denotes the proton gyro-frequency). Second, the spectrum is integrated over the frequencies to obtain the spectrum in the three-dimensional wavevector domain (bottom panels in Fig. 5). The fluctuation energy peaks in the positive $z$ direction, indicating that the fluctuations propagate toward the center of the plasma sheet (northward propagation in the southern outer sheet) with a moderate inclination to sunward and duskward.

The spectrum is also visualized in the mean-fieldaligned (MFA) system in Fig. 6, spanning the mean magnetic field direction (the parallel direction) and the flow direction (in the perpendicular-1 and parallel plane) in the same panel style as Fig. 5. Again, there is no clear organization in the wavenumber-frequency diagram (top panels). The wavevector spectrum in the MFA coordinate system indicates that a large amount of the fluctuation 


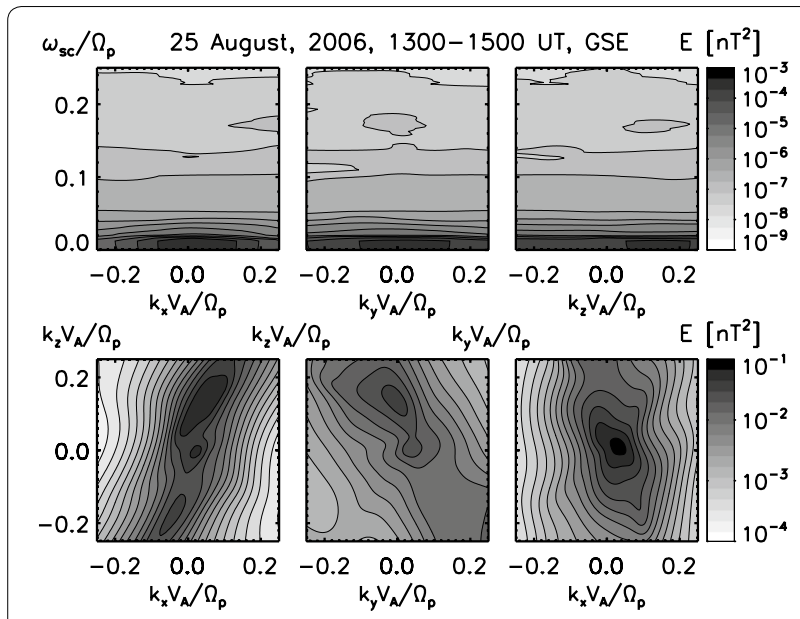

Fig. 5 Four-dimensional magnetic energy spectrum projected onto three planes spanning the wavevector components and the spacecraft-frame frequencies in the GSE coordinate system by averaging over the wavevector components orthogonal to the planes (top panels), and that projected onto the three wavevector planes by integrating over the frequencies and averaging over the wavevector components. The wavevectors and the frequencies are normalized to the ion inertial length and the proton gyro-frequency, respectively

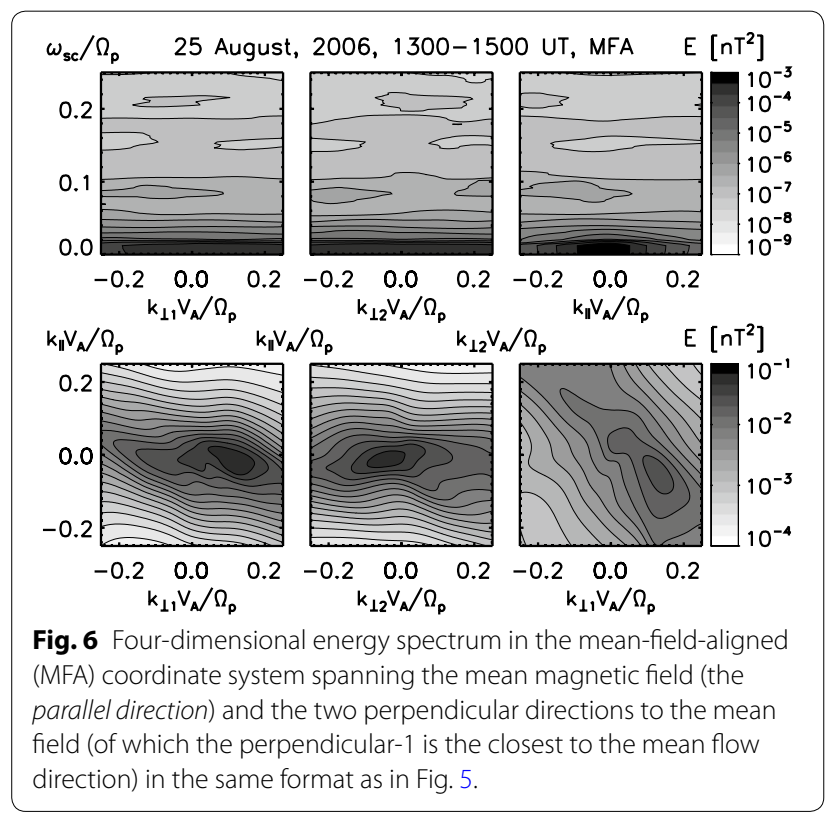

energy is associated with the wavevectors that are markedly perpendicular to the mean magnetic field and in the nearly streamwise sense (in the perpendicular-1 direction). It is interesting to observe that the gyrotropy (axial symmetry around the mean magnetic field) is broken. The spectral energy shows an extension in the oblique direction from the flow direction. This direction happens to be the sunward and northward (toward the center of the plasma sheet) in the GSE coordinate representation (see Fig. 5).

The interpretation of the perpendicular extension of the wave energy spectrum as an erroneous estimate of the wave propagation direction due to the group speed interference as discussed in the chorus wave study (Goldstein and Tsurutani 1984) is worthwhile for further studies involving multi-point measurement techniques, since neither MSR nor the method by Goldstein and Tsurutani (1984) assumes one wave at one frequency and can identify individual wave vectors by modeling the measured fluctuations as a set of unspecified discrete waves and isotropic noise at each frequency.

\section{Dispersion relation diagram}

To further verify the existence of the linear wave modes, the coherent wave components are studied using the dispersion relation diagram and the phase speed diagram for comparison with the MHD linear-mode waves. The dispersion relation diagram presents the distribution of frequencies and the wavevector angles from the mean magnetic field for the local spectral peaks as a function of the wavenumbers (Fig. 7). The dotted line in the wavenumber-frequency diagram (top panel) represents a reference dispersion relation. Field-aligned Alfvén waves with the relation $\omega=k V_{\mathrm{A}}$ are taken for the reference dispersion relation. The Alfvén speed is estimated around $V_{\mathrm{A}}=1390.6 \mathrm{~km} / \mathrm{s}$. The Doppler shift (which is at most a few hundreds of $\mathrm{km} / \mathrm{s}$, see Fig. 2) is not significant as the flow speed is sufficiently below the Alfvén speed. The coherent waves show both higher and lower frequencies than that of the Alfvén waves (on the reference dispersion relation in the top panel), and the wavevector angles

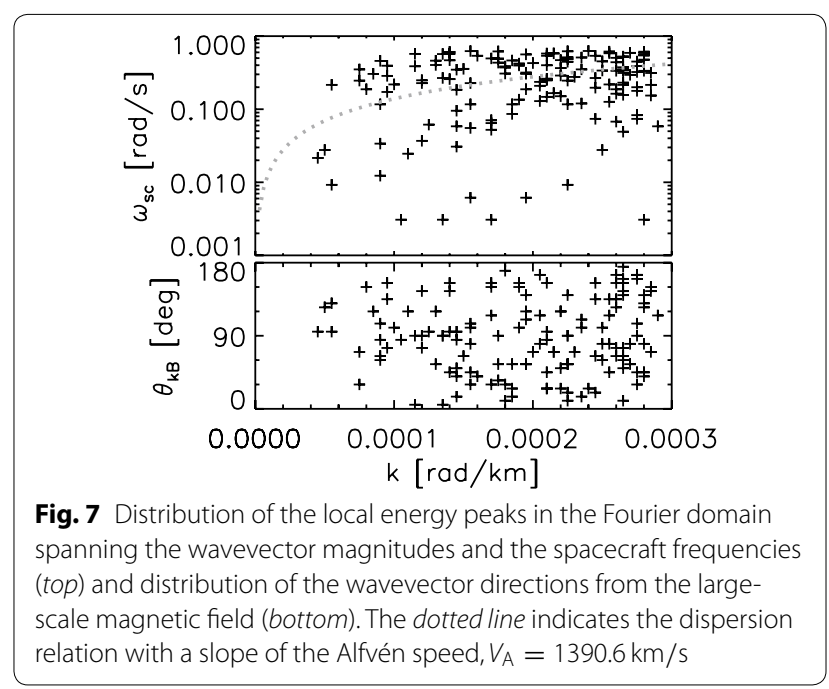


are broadly distributed including quasi-parallel, oblique, quasi-perpendicular, and quasi-anti-parallel directions with respect to the mean magnetic field (bottom panel).

It is unlikely that kinetic modes are present besides MHD modes because even kinetic modes show a systematic organization of the wave data in the dispersion relation diagram. For quasi-perpendicular wavevectors (with respect to the mean magnetic field), the candidate kinetic-wave modes for a Maxwellian velocity distribution function on the spatial scales of interest $(20 \%$ of ion inertial wavenumber) are only whistlers and ion Bernstein modes (both of which become the fast magnetosonic mode in MHD), kinetic Alfvén (which becomes the obliquely propagating Alfvén mode in MHD), and kinetic slow modes (which becomes the slow mode in MHD). Another possibility that the beam resonant mode might exist is again unlikely because the beam resonant mode has a quasi-parallel propagation.

\section{Phase speed diagram}

The phase speed diagram is presented in Fig. 8. The phase speeds of the detected coherent waves are determined using the frequencies and the wavevectors as a vectorial quantity as $\vec{v}_{\mathrm{ph}}=\frac{\omega}{k} \frac{k}{k}$, where $k=|\vec{k}|$, and are projected onto a two-dimensional plane spanning the phase speeds parallel and perpendicular to the mean magnetic field by multiplying the sign of the sine of the azimuthal angle measured from the projection of the flow direction onto the perpendicular plane (in the direction to $(\vec{B} \times \vec{U}) \times \vec{B}$ ) as

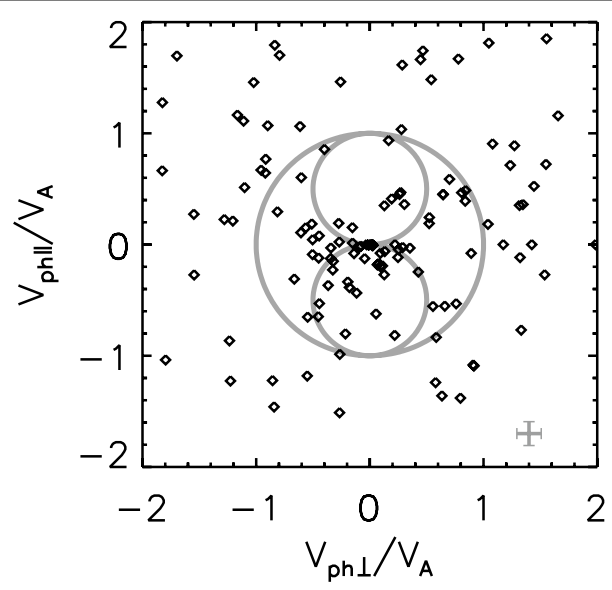

Fig. 8 Phase speed diagram of the observed fluctuations as a function of the phase speeds parallel (the vertical axis) and perpendicular (the horizontal axis) to the large-scale magnetic field. The phase speeds are normalized to the Alfvén speed. The gray curves indicate the phase speeds of the Alfvén mode (inner circles) and the fast most (outer circle). The error bars are shown at the bottom right corner, representing the flow speed variation

$$
\left(v_{\mathrm{ph} \|}, v_{\mathrm{ph} \perp}\right)=\left(v_{\mathrm{ph}} \cos \theta, v_{\mathrm{ph}} \sin \theta \operatorname{sgn}(\sin \phi)\right) .
$$

where $\operatorname{sgn}(\sin \phi)=\sin \phi /|\sin \phi|$. In other words, positive and negative values in $v_{\mathrm{ph} \perp}$ have the sense of the flow direction and its opposite direction, respectively. The phase speeds are normalized to the Alfvén speed. The diagram is compared with the phase speeds of the Alfvén mode (the inner circles in gray) and the fast magnetosonic mode (the outer circle in gray). The phase speeds of the coherent waves do not agree with that of the MHD linearmode waves. Both the directions and the magnitudes of the phase speeds are broadly distributed. A reasonably good agreement with the MHD linear-mode can be found only for the coherent waves with small phase speeds (below the Alfvén speed) propagating in quasi-perpendicular directions to the mean magnetic field. Errors in the phase speed estimate mostly come from the flow velocity fluctuations, which causes Doppler shift and broadening of frequencies (shown as a cross at the bottom right corner in Fig. 8). The errors are not significant because the flow velocities are well below the Alfvén speed. The phase speed diagram indicates that the coherent wave components contain both linear-mode fluctuations and nonlinear ones appearing as sideband or nonlinear waves.

\section{Conclusion and discussion}

The picture of linear modes does not hold even if the amplitude appears to be sufficiently small, $\delta B / B_{0} \simeq 1 / 16$. Rather, turbulent fluctuations in magnetohydrodynamics should be regarded as a set sideband waves that have frequencies off from the linear modes. For incoherent waves, the energy spectrum is anisotropic and exhibits an extension in the quasi-perpendicular direction to the large-scale magnetic field. The axial symmetry of the energy spectrum around the large-scale field is broken, and the spectrum is extended in the direction from the southern lobe to the plasma sheet center (Fig. 9). The fluctuation energy is large at very low frequencies and

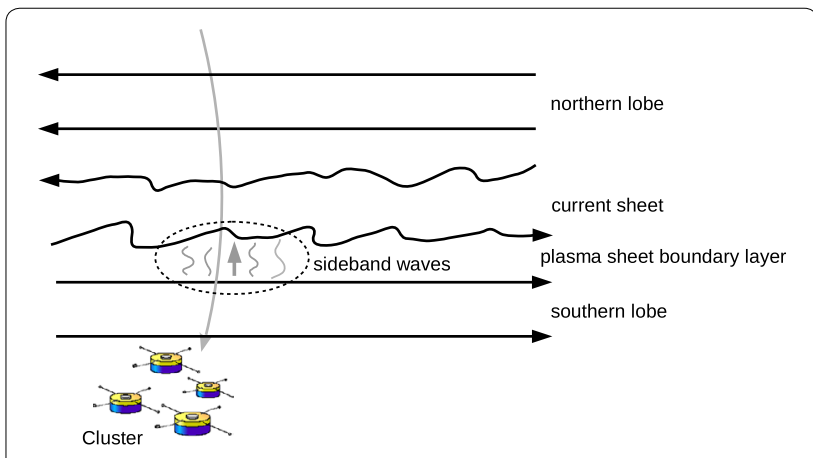

Fig. 9 Schematic view of MHD turbulence with sideband waves propagating toward the center of plasma sheet in the magnetotail 
decays monotonously toward higher frequencies irrespective of the wavevector directions. The spectrum in the wavenumber-frequency domain shows no clear organization of dispersion relation. Perhaps the distinct linear modes may exist near the source and they have decayed into turbulence before observed by Cluster. However, this statement remains only speculative.

For coherent waves, the dispersion relation diagram and the phase speed diagram do not show a positive evidence for the existence of the linear modes in magnetohydrodynamics. The wavevector distribution is nearly isotropic and the average phase speed is of the order of the Alfvén speed. Yet, there is a weak indication of the linear MHD modes in the quasi-perpendicular directions.

It is worth noting that there are many possibilities for a Kolmogorov-type spectrum, e.g., eddies splitting, Alfvén wave scattering when combined with the critical balance model (Goldreich and Sridhar 1995; Tsurutani et al. 1995). Kinetic-wave mode interpretation is in principle possible, but kinetic-wave modes such as whistler and beam resonant modes appear as particularly clear dispersion branches (Narita et al. 2003), which is not the case in the obtained results. Also, the spatial scales of the wave observation are below the ion kinetic one. However, a solid answer needs a further study involving full kinetic instability analysis with a proper measurement of velocity distribution functions such as a bump-on-tail shape.

Appearance of higher frequencies than that of the linear modes can be interpreted as cascading three or multiple wave interactions using the matching conditions for frequencies $\left(\omega_{1}+\omega_{2}=\omega_{3}+\cdots\right)$ and wavevectors $\left(\vec{k}_{1}+\vec{k}_{2}=\vec{k}_{3}+\cdots\right)$ in a perturbation way. For example, one may construct a scenario such that a forward-propagating Alfvén wave (e.g., with respect to the large-scale magnetic field) interacts with a backward propagating slow mode, generating sideband waves at a low wavenumber and a higher frequency. This type of interaction explains the fluctuations at higher frequencies. One may also couple the Alfvén wave with the zero-frequency mode (which represents a standing wave) to generate the sideband waves at low frequencies and high wavenumbers. The sideband waves cannot survive for a long time, but can interact with the other wave components (both linear-mode and sideband waves) within the lifetime to generate sideband waves even at higher frequencies or wavenumbers. Such wave nonlinearities or wave-wave couplings can be tested using higher-order statistics such as bispectrum for three-wave interactions. Fortunately, the bispectral method is applicable in both the frequency and the wavevector domain using the four-spacecraft data and will be a target of intensive studies to reveal the detailed fluctuation processes in MHD turbulence.
Three-wave resonance has in fact been observed partially. For example, the frequency matching was successfully tested by Spangler et al. (1997), and the parallel wavenumber matching has also successfully been tested by Narita et al. (2008). On the other hand, in most of observations, wave-wave coupling process has not been observed in space plasmas probably because the plasma does not have enough time to develop into turbulence through wave-wave couplings (Tsurutani et al. 1997). Determination of the wave helicity will help to uniquely identify the type of wave-wave coupling. The wave helicity is measurable using four-point magnetic field data (Narita et al. 2009). A search for simultaneous matching of both frequencies and wavevectors is an important subject and still needs to be done, which requires a more sophisticated analysis method using multi-spacecraft data.

A possible scenario is that sideband wave formation plays an important role in MHD turbulence and is motivated by the recent observational confirmation about the existence of sideband waves adjacent to the linearmode branch in solar wind turbulence (Perschke et al. 2013, 2014) Energy transport in the frequency domain represents generation of sideband waves. For quasi-perpendicular propagations, the fast mode has finite frequencies, while the other two MHD wave modes, Alfvèn and slow, have zero frequency. Sideband waves develop in such a way that the energy stored along the linear-mode branches flows toward sideband waves. The wave study shown in this manuscript indicates that it is unlikely to excite sideband waves toward higher frequencies. The energy transport in the frequency domain is slower than the transport in the wavenumber domain. Therefore, most of the fluctuation energies is constrained to lower frequencies, e.g., up to the frequencies of the fast mode. The sideband formation influences the wavevector anisotropy, as well. The observational result that most of the fluctuation energies are associated with quasi-perpendicular wavevectors to the mean magnetic field indicates a scenario that the sideband wave formation is not an isotropic process but anisotropic, presumably because the zero-frequency or nearly zero-frequency mode as realized by the perpendicular wavevector limit of the Alfvén and the slow modes are more essential in MHD turbulence. The wave evolution scenario into turbulence is illustrated in Fig. 10.

It is also important to estimate turbulent transports such as the turbulent magnetic diffusivity, evaluation of timescale as well as the energy spectra (in the wavevector domain) from the observations. Yet, to the author's knowledge, there is no established method available to directly determine the diffusivity or timescale. For example, the wavevector anisotropy or the shape of the 


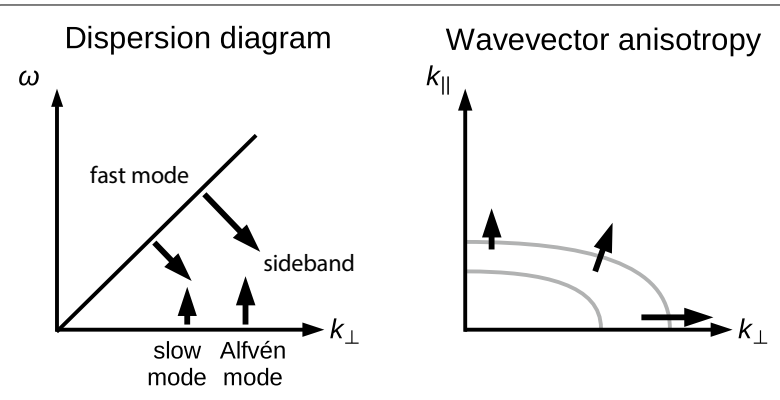

Fig. 10 Energy cascade model in the wavenumber-frequency domain (dispersion diagram, left panel) and the wavevector domain (wavevector anisotropy, right panel)

wavevector spectrum for MHD turbulence is still one of the actively studied topics, e.g., Narita (2015). Such a task requires a construction of a proper model (called the estimator) to derive the physically relevant quantities from the observation and will be a subject of intensity study.

\section{Author details}

${ }^{1}$ Space Research Institute, Austrian Academy of Sciences, Schmiedlstraße 6, 8042 Graz, Austria. ${ }^{2}$ Institut für Geophysik und extraterrestrische Physik, Technische Universität Braunschweig, Mendelssohnstraße 3, 38106 Braunschweig, Germany.

\section{Acknowledgements}

The author thanks Rumi Nakamura and Zoltan Vörös for suggestions and discussions during the manuscript preparation. The MSR algorithm was developed through the collaboration with Uwe Motschmann and Karl-Heinz Glassmeier at Technische Universität Braunschweig, Germany. Cluster FGM and CIS teams as well as Cluster Science Archive are acknowledged for providing the data for this study. AE index (hourly average, provisional data) was provided by World Data Center for Geomagnetism, Kyoto, Japan.

\section{Competing interests}

The authors declare that they have no competing interests.

Received: 24 November 2015 Accepted: 13 April 2016

Published online: 27 April 2016

\section{References}

Balogh A, Carr CM, Acuña MH, Dunlop MW, Beek TJ, Brown P, Fornaçon K-H, Georgescu E, Glassmeier K-H, Harris J, Musmann G, Oddy T, Schwingenschuh K (2001) The Cluster magnetic field investigation: overview of in-flight performance and initial results. Ann Geophys 19:1207-1217. doi:10.5194/angeo-19-1207-2001

Baumjohann W, Paschmann G, Cattell CA (1989) Average plasma properties in the central plasma sheet. J Geophys Res 94:6597-6606. doi:10.1029/ JA094iA06p06597

Capon J (1969) High resolution frequency-wavenumber spectrum analysis. Proc IEEE 57:1408-1418. doi:10.1109/PROC.1969.7278

Choi J, Song I, Kim HM (1993) On estimating the direction of arrival when the number of signal sources is unknown. Signal Process 34:193-205. doi:10.1016/0165-1684(93)90162-4

Cowley SWH, Hynds RJ, Richardson IG, Daly PW, Sanderson TR, Wenzel K-P, Slavin JA, Tsurutain BT (1984) Energetic ion regimes in the deep geomagnetic tail: ISEE-3. Geophys Res Lett 11:275-278. doi:10.1029/ GL 011 i003p00275

Escoubet CP, Fehringer M, Goldstein M (2001) The Cluster mission. Ann Geophys 19:1197-1200. doi:10.5194/angeo-19-1197-2001
Gary SP, Madland CD, Tsurutani BT (1985) Electromagnetic ion beam instabilities: Il. Phys Fluids 28:3691-3695. doi:10.1063/1.865101

Glassmeier K-H, Motschmann U, Dunlop M, Balogh A, Acuña MH, Carr C, Musmann G, Fornaon K-H, Schweda K, Vogt J, Georgescu E, Buchert S (2001) Cluster as a wave telescope-first results from the fluxgate magnetometer. Ann Geophys 19:1439-1447. doi:10.5194/angeo-19-1439-2001 (correction in 21, 1071 2003)

Goldreich P, Sridhar S (1995) Toward a theory of interstellar turbulence. 2: strong alfvenic turbulence. Astrophys J 438:763-775. doi:10.1086/175121

Goldreich P, Sridhar S (1997) Magnetohydrodynamic turbulence revisited. Astrophys J 485:680-688. doi:10.1086/304442

Goldstein BE, Tsurutani BT (1984) Wave normal directions of chorus near the equatorial source region. J Geophys Res 89:2789-2810. doi:10.1029/ JA089iA05p02789

Iroshnikov PS (1964) Turbulence of a conducting fluid in a strong magnetic field. Sov Astron 7:566-571

Kaburaki O, Uchida Y (1971) Magnetohydrodynamic wave-mode coupling. Quantum field-theoretical approach to weakly non-linear case with application to solar coronal heating. Publ Astron Soc Japan 23:405-423

Kraichnan R (1965) Inertial-range spectrum of hydromagnetic turbulence. Phys Fluids 8:1385-1387. doi:10.1063/1.1761412

Motschmann U, Woodward TI, Glassmeier K-H, Southwood DJ (1995) Wave field analysis by magnetic measurements at satellite arrays: generalized minimum variance analysis. Adv Space Res 18:315-319. doi:10.1016/0273-1177(95)00964-7

Motschmann U, Woodward TI, Glassmeier KH, Southwood DJ, Pinçon JL (1996) Wavelength and direction filtering by magnetic measurements at satellite arrays: generalized minimum variance analysis. J Geophys Res 101:4961-4966. doi:10.1029/95JA03471

Narita Y, Glassmeier K-H, Schäfer S, Motschmann U, Sauer K, Dandouras I, Fornaçon K-H, Georgescu E, Rème H (2003) Dispersion analysis of ULF waves in the foreshock using cluster data and the wave telescope technique. Geophys Res Lett 30:SSC 43-€œ1. doi:10.1029/2003GL 017432

Narita Y, Glassmeier K-H, Décréau P, Hada T, Motschmann U, Nariyuki Y (2008) Evaluation of bispectrum in the wave number domain based on multi-point measurements. Ann Geophys 26:3389-3393. doi:10.5194/ angeo-26-3389-2008

Narita Y, Kleindienst G, Glassmeier K-H (2009) Evaluation of magnetic helicity density in the wave number domain using multi-point measurements in space. Ann Geophys 27:3967-3976. doi:10.5194/angeo-27-3967-2009

Narita Y, Glassmeier K-H, Motschmann U (2011) High-resolution wave number spectrum using multi-point measurements in space-the multi-point signal resonator (MSR) technique. Ann Geophys 29:351-360. doi:10.5194/ angeo-29-351-2011

Narita Y (2015) Non-elliptic wavevector anisotropy for magnetohydrodynamic turbulence. Ann Geophys 33:1413-1419. doi:10.5194/ angeo-33-1413-2015

Neubauer FM, Glassmeier K-H (1990) Use of an array of satellites as a wave telescope. J Geophys Res 95(19):115-122. doi:10.1029/JA095iA11 p19115

Perschke C, Narita Y, Gary SP, Motschmann U, Glassmeier K-H (2013) Dispersion relation analysis of turbulent magnetic field fluctuations in fast solar wind. Ann Geophys 31:1949-1955. doi:10.5194/angeo-31-1949-2013

Perschke C, Narita Y, Motschmann U, Glassmeier K-H (2014) Multi-spacecraft bservations of linear modes and sideband waves in ion-scale solar wind turbulence. Astrophys J Lett 793:L25. doi:10.1088/2041-8205/793/2/L25

Pinçon JL, Lefeuvre F (1991) Local characterization of homogeneous turbulence in a space plasma from simultaneous measurements of field components at several points in space. J Geophys Res 96:1789-1802. doi:10.1029/90JA02183

Rème H, Aoustin C, Bosqued JM, Dandouras I et al (2001) First multispacecraft ion measurements in and near the Earth's magnetosphere with the identical Cluster ion spectrometry (CIS) experiment. Ann Geophys 19:1303-1354. doi:10.5194/angeo-19-1303-2001

Robert P, Roux A, Harvey CC, Dunlop MW, Daly PW, Glassmeier K-H (2001) Tetrahedron geometric factors. In: Paschmann G, Daly PW (eds) Analysis methods for multi-spacecraft sata. ISSI Scientific Report SR-001, pp 323-348

Runov A, Sergeev VA, Nakamura R, Baumjohann W, Apatenkov S, Asano Y, Takada T, Volwerk M, Vörös Z, Zhang TL, Sauvaud J-A, Rème H, Balogh A (2006) Local structure of the magnetotail current sheet: 2001 Cluster observations. Ann Geophys 24:247-262. doi:10.5194/angeo-24-247-2006 
Schmidt RO (1986) Multiple emitter location and signal parameter estimation. IEEE Trans Antennas Propag AP-34:276-280. doi:10.1109/ TAP.1986.1143830

Smith EJ, Tsurutani BT, Rosenberg RL (1978) Observations of the interplanetary sector structure up to heliographic latitudes of $16^{\circ}$ : Pioneer 11. J Geophys Res Space Phys 83:717-724. doi:10.1029/JA083iA02p00717

Spangler SR, Leckband JA, Cairns IH (1997) Observations of the parametric decay instability of nonlinear magnetohydrodynamic waves. Phys Plasmas 4:846-855. doi:10.1063/1.872183

Storey LRO, Lefeuvre F (1979) The analysis of 6-component measurements of a random electromagnetic wave field in a magnetoplasma-I. The direct problem. Geophys J R Astron Soc (Geophys J Int) 56:255-269. doi:10.1111/j.1365-246X.1979.tb00163.x

Storey LRO, Lefeuvre F (1980) The analysis of 6-component measurements of a random electromagnetic wave field in a magnetoplasma-II. The integral kernels. Geophys J R Astron Soc (Geophys J Int) 62:173-194. doi:10.1111/ j.1365-246X.1980.tb04850.x

Tsurutani BT, Slavin JA, Smith EJ, Okida R, Jones DE (1984a) Magnetic structures of the distant geotail from -60 to -200 Re: ISEE-3. Geophys Res Lett 11:1-4. doi:10.1029/GL011i001p00001

Tsurutani BT, Jones DE, Slavin JA, Sibeck DG, Smith EJ (1984b) Plasmasheet magnetic fields in the distant tail. Geophys Res Lett 11:1062-1065. doi:10.1029/GL011i010p01062

Tsurutani BT, Jones DE, Sibeck DG (1984c) The two-lobe structure of the distant ( $X \geq 200 \mathrm{Re}$ ) magnetotail. Geophys Res Lett 11:1066-1069. doi:10.1029/GL011i010p01066

Tsurutani BT, Smith EJ (1984) Magnetosonic waves adjacent to the plasma sheet in the distant magnetotail: ISEE-3. Geophys Res Lett 11:331-334 doi:10.1029/GL011i004p00331

Tsurutani BT, Richardson IG, Thorne RM, Butler W, Smith EJ, Cowley SWH, Gary SP, Akasofu S-I, Zwickl RD (1985) Observations of the right-hand resonant ion beam instability in the distant plasma sheet boundary layer. J Geophys Res 90:12159-12172. doi:10.1029/JA090iA12p12159

Tsurutani BT, Goldstein BE, Burton ME, Jones DE (1986) A review of the ISEE-3 geotail magnetic field results. Planet Space Sci 34:931-960. doi:10.1016/0032-0633(86)90004-8
Tsurutani BT, Burton ME, Smith EJ, Jones DE (1987) Statistical properties of magnetic field fluctuations in the distant plasmasheet. Planet Space Sci 35.289-293 doi:10.1016/0032-0633(87)90155-3

Tsurutani BT, Glassmeier K-H, Neubauer FM (1995) An intercomparison of plasma turbulence at three comets: Grigg-Skjellerup, Giacobini-Zinner, and Halley. Geophys Res Lett 22:1149-1152. doi:10.1029/95GL00806

Tsurutani BT, Glassmeier K-H, Neubauer FM (1997) A review of nonlinear low frequency (LF) wave observations in space plasmas: on the development of plasma turbulence. In: Hada T, Matsumoto H (eds) Nonlinear waves and chaos in space plasmas. Terra Scientific Publishing (TERRAPUB), Tokyo, pp 1-44

Verma MK (2004) Statistical theory of magnetohydrodynamic turbulence: recent results. Phys Rep 401:229-380. doi:10.1016/j.physrep.2004.07.007 (correction 422, 223-224, doi:10.1016/j.physrep.2005.10.004, 2006)

Vörös Z, Baumjohann W, Nakamura R, Volwerk M, Runov A, Zhang TL, Eichelberger HU, Treumann R, Georgescu E, Balogh A, Klecker B, Rème H (2004) Magnetic turbulence in the plasma sheet. J Geophys Res 109:A11215. doi :10.1029/2004JA010404

Vörös Z, Baumjohann W, Nakamura R, Runov A, Volwerk M, Takada T, Lucek EA, Rème H (2007a) Spatial structure of plasma flow associated turbulence in the Earth's plasma sheet. Ann Geophys 25:13-17. doi:10.5194/ angeo-25-13-2007

Vörös Z, Baumjohann W, Nakamura R, Runov A, Volwerk M, Asano Y, Jankovičová D, Lucek EA, Rème H (2007b) Spectral scaling in the turbulent Earth's plasma sheet revisited. Nonlinear Process Geophys 14:535-541. doi:10.5194/npg-14-535-2007

von Stein R, Glassmeier K-H, Dunlop M (1992) A configuration parameter for the Cluster satellites. Tech. Rep. 2/1992, Institut für Geophysik und Meteorologie der Technischen Universität Braunschweig

Winterhalter D, Smith EJ, Burton ME, Murphy N, McComas DJ (1994) The heliospheric plasma sheet. J Geophys Res 99:6667-6680. doi:10.1029/93JA03481

Yokoi N, Rubinstein R, Yoshizawa A, Hamba F (2008) A turbulence model for magnetohydrodynamic plasmas. J Turbul 9:N37. doi:10.1080/14685240802433057

\section{Submit your manuscript to a SpringerOpen ${ }^{\circ}$ journal and benefit from:}

- Convenient online submission

- Rigorous peer review

- Immediate publication on acceptance

- Open access: articles freely available online

- High visibility within the field

- Retaining the copyright to your article

Submit your next manuscript at springeropen.com 